\title{
Prediction of a moving target's position in fast goal-directed action
}

\author{
Jeroen B. J. Smeets, Eli Brenner \\ Vakgroep Fysiologie, Erasmus Universiteit Rotterdam, Postbus 1738, NL-3000 DR Rotterdam, The Netherlands
}

Received: 10 February 1995/Accepted in revised form: 30 May 1995

\begin{abstract}
Subjects made fast goal-directed arm movements towards moving targets. In some cases, the perceived direction of target motion was manipulated by moving the background. By comparing the trajectories towards moving targets with those towards static targets, we determined the position towards which subjects were aiming at movement onset. We showed that this position was an extrapolation in the target's perceived direction from its position at that moment using its perceived direction of motion. If subjects were to continue to extrapolate in the perceived direction of target motion from the position at which they perceive the target at each instant, the error would decrease during the movements. By analysing the differences between subjects' arm movements towards targets moving in different (apparent) directions with a linear second-order model, we show that the reduction in the error that this predicts is not enough to explain how subjects compensate for their initial misjudgements.
\end{abstract}

\section{Introduction}

Due to delays in the nervous system, and to the inertial and viscous properties of our bodies, a neural command to move our hand somewhere does not lead to an instantaneous displacement of the hand to that position. If the hand is to hit or grasp a moving object, it is therefore a good strategy to aim at a future position of the object. This paper studies whether visual information about the direction of motion is used to predict this future position, and how this position is updated during the movement.

For making movements in general, both the 'when' and the 'where' of an action are important. In this study, we focus on the visual information that is used to determine the 'where' of the action. For a time-constrained action towards a moving object, the position of the contact has to be determined from visual information. This could be done by extrapolating the trajectory using the speed of the object. We recently showed (Brenner and

Correspondence to: J. B. J. Smeets
Smeets 1994a; Smeets and Brenner 1995) that subjects do not use the perceived speed to predict a future position of an object. Instead, subjects constantly extrapolate from the perceived position using a default speed. As they continuously update their prediction, the systematic error in extrapolating the future position of the target decreases with time to contact. Subjects compensate for the remaining error by gearing the speed of their action to the perceived speed of the object (Brenner and Smeets 1994b). Subjects thus gear their action to two different perceptual variables: the perceived position of an object determines the hand's trajectory, whereas the perceived speed determines the timing of the movement. This finding contrasts with the hypothesis that subjects extract one single invariant (for instance $\tau$ ) from the sensory information, and gear all aspects of their action to that invariant (Lee and Young 1986; Peper et al. 1994).

For predicting an object's future position, both its speed and the direction of its motion have to be known. The first question addressed in this study is whether subjects ignore the perceived direction of motion in the same way they ignore the target's speed. If not, how is the perceived direction of motion used when making goaldirected movements towards moving targets?

To investigate the use of visual information in motor control, several aspects of movements can be analysed. The beginning of the movement can show how the information is used in planning the movement. The final result can reveal whether the information is required for fulfilling the task. A comparison between the start and the final result can reveal whether information is used during the movement. Our approach is to investigate how the information is used by analysing the shape of the trajectories.

The shape of the trajectories is determined by an interaction between information about target position (translated into a 'motor program') and the biomechanics of the arm. As the equations leading from muscle activation to movement of the arm are highly non-linear, modelling the biomechanics is a difficult approach. Van Sonderen and Denier van der Gon (1990) have shown that such a model is feasible for describing responses to large target displacements. However, due to the uncertainty of many parameters and assumptions in their 
model, it is not suitable for studying the effects of small changes in visual information. We will therefore not attempt to model the complete arm movements.

As we are mainly interested in the use of visual information for the fine-tuning of movements, we adopt a different approach. Our approach is based on the fact that any non-linear system without discontinuities can be approximated by a linear system as long as it is only used for describing the effect of small changes in some of the relevant variables (Taylor's Theorem). In our experiment, we are dealing with small (about $5 \mathrm{~cm}$ ) variations in arm movements (total amplitude is more than $35 \mathrm{~cm}$ ). The differences between these slightly different movements are considered to be governed by a linear system. Differences of the same magnitude were successfully modelled in this manner in a previous study (Smeets and Brenner 1995). The main advantage of using such a model is that it enables us to estimate the position that a subject is aiming at, at each moment during the movement.

The present paper has two goals. The first, more specific goal is to investigate whether the direction of a target's motion is used in predicting its future position and, if so, whether the direction used in motor control is the direction subjects perceive. The second, more general goal is to provide a first step towards a description of how continuous visual information is used in guiding arm movements.

\section{Methods}

\subsection{Experimental approach}

We performed three experiments to evaluate the use of visual information in guiding arm movements. To distinguish between information on position and direction of motion of the target, motion of the background was used. Background motion is known to influence the perceived direction of motion. In all three experiments the background moved in about $40 \%$ of the trials. Subjects could not predict whether the background would move in individual trials. In the first two experiments, subjects were asked to reproduce some aspects of the visual information: the perceived direction of target motion and the target's final perceived position. In the third experiment, subjects had to move their arm as quickly as possible to hit similar moving targets.

\subsection{Subjects}

Ten right-handed volunteers from our department participated in the experiment (including the authors). The subjects were all familiar with the experimental protocol, but were (except for the authors) naive with respect to the exact purpose of the experiment. All subjects completed the set of experiments within $1 \mathrm{~h}$.

\subsection{Experimental setup}

The setup was designed to leave the subjects totally unrestrained in their movements, while maintaining total

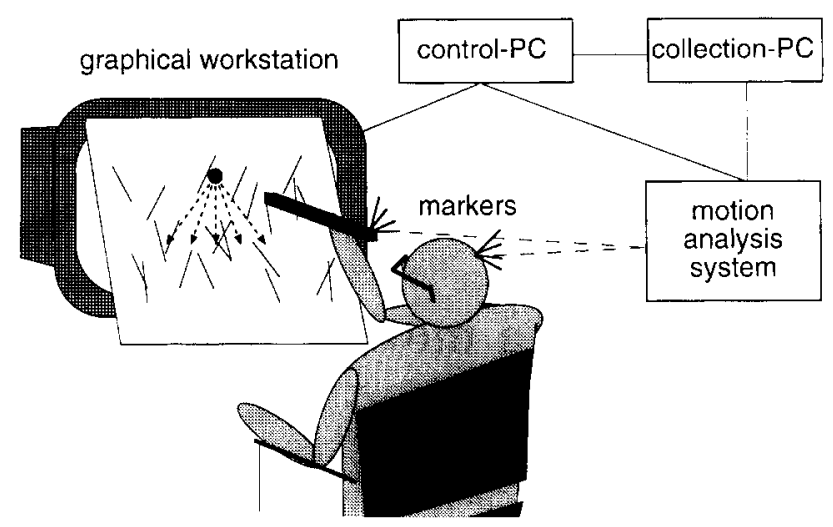

Fig. 1. Overview of the experimental setup. Subjects were free to choose their position relative to the screen, as long as they kept the rod within $5 \mathrm{~cm}$ of a position $40 \mathrm{~cm}$ in front of the screen. The subjects mainly moved their hands within a horizontal plane

control of the visual information. The equipment had to perform two tasks simultaneously: to generate images and to record the subject's movements. Each task was performed by a dedicated machine. A control PC regulated the course of the experiment and the exchange of essential information between the other machines. An overview of the experimental setup is given in Fig. 1. Subjects sat in an adjustable chair in front of a computer screen in a dark room. They were allowed to position the chair so that they could comfortably view the screen and move their arm. They could not see their hand, nor had they any visual points of reference other than those presented on the workstation.

Images were generated on a Unix-based graphical workstation (Silicon Graphics). The monitor of the system was protected by a transparent screen, oriented at $30^{\circ}$ to the vertical. The images that were presented were made to appear to lie on this screen with the aid of LCD shutter spectacles (Silicon Graphics) and by accounting for motion parallax (see below). Each image consisted of a simulated background of about 40 yellow lines (simulated line length $4 \mathrm{~cm}$, random orientation between $-60^{\circ}$ and $60^{\circ}$ around the vertical; within an area of $18 \times 15 \mathrm{~cm})$ and a red disk $(1 \mathrm{~cm}$ radius $)$ that floated across this background. The background could move horizontally at $9 \mathrm{~cm} / \mathrm{s}$ in either direction, or remain static. To mask the appearance and disappearance of lines at the borders of the background (when it moved), the intensity of the lines faded in the leftmost and rightmost $4 \mathrm{~cm}$. The disk always moved downwards at $12 \mathrm{~cm} / \mathrm{s}$. In addition, it could move horizontally at 4.5 or $9 \mathrm{~cm} / \mathrm{s}$ in either direction. This resulted in motion in five directions between $-37^{\circ}$ and $37^{\circ}$ from vertical.

Movements of the subjects' hand and head were recorded by a motion analysis system based on active infrared markers (Optotrak 3010, Northern Digital). The markers for measuring movements of the hand were attached to a Perspex rod ( $22 \mathrm{~cm}$ long, $1 \mathrm{~cm}$ radius) held by the subject; those for measuring head movements were attached to the LCD spectacles. The resolution of the position measurement was better than $0.1 \mathrm{~mm}$ in all three 
dimensions. Position data were collected by a PC (collection PC) at a frame rate of $300 \mathrm{~Hz}$ for $1.5 \mathrm{~s}$ per trial (software from Northern Digital).

The control PC controlled the generation of the images and the collection of the data by the collection PC. It calculated the positions of the subject's eyes from the markers' positions, and sent these data to the graphical workstation so that motion parallax could be accounted for in the images. It also calculated the position of the tip of the rod (in the text, we will sometimes use 'position of the hand' to indicate this position) from the markers' positions, and sent messages to the graphical workstation indicating what was to be presented. The software in the graphical workstation and in the control PC was home-built.

\subsection{Protocol of the two perception experiments}

Each subject participated in two perception experiments: one examining the perceived direction of target motion, the other examining the perceived position of the target.

Disks appeared approximately $1 \mathrm{~cm}$ above the centre of the screen (the exact position varied at random within $1 \mathrm{~cm}$ in both horizontal and vertical directions) and moved downwards in one of the five directions. After $367 \mathrm{~ms}$, the disk was removed from the screen. Only the background was visible during the next $500 \mathrm{~ms}$. Then, the background was replaced and a stationary disk appeared within $1 \mathrm{~cm}$ (in a random direction) of the position at which the disk had disappeared. In the position experiment, subjects were asked to move the stationary disk (the position of which was coupled to that of the computer mouse) to the position at which the moving disk had disappeared. In the direction experiment, a green line was drawn on the stationary disk. Subjects were asked to orient this line (the orientation of which was coupled to the computer mouse) so that it aligned with the perceived direction of motion.

All five directions of target motion were presented on a static background. To examine the influence of background motion, four conditions were included in which the background moved for as long as the disk was presented and during the $500 \mathrm{~ms}$ thereafter. The conditions were chosen to provide a good comparison between trials with and without background motion for effects up to $100 \%$. Each condition was repeated five times, yielding a total of 45 trials for each experiment.

\subsection{Protocol of the motor experiment}

Subjects held the Perspex rod with their right hand and looked at the background on the screen. The background disappeared from view only when it was replaced by messages about the experiment. These messages guided the subject's hand to within $5 \mathrm{~cm}$ of a point $40 \mathrm{~cm}$ from the centre of the screen. Subjects had no visual references other than the stimulus on the screen.

Subjects positioned the chair so that they were comfortable. This usually meant that they started the movements with an almost fully flexed elbow and both upper and lower arm more or less parallel to their trunk. They could not see their hand, although they could deduce its approximate position from the occlusion of the background once the hand was near the screen. Disks could appear at the top of the screen moving downwards in one of five directions, or at rest in positions near the lower edge of the screen. The position at which the disks appeared was defined with respect to the actual position of the hand; it therefore varied relative to the background on the screen. Data collection started at the moment the disk appeared.

Subjects were instructed to hit the disk with the Perspex rod as quickly as possible. If the centre of the rod was within $1.8 \mathrm{~cm}$ of the centre of the disk when the screen was hit, the disk stopped moving and was displayed as broken into four unequal pieces. If the subject hit a position the disk had passed, the disk kept moving in the same direction; if he or she hit ahead of the disk, it bounced back in the opposite direction. Subjects were instructed to move both accurately (to hit the disk) and quickly: the sum of the reaction time $(R T)$ and movement time $(M T)$ was to be minimized.

In addition to the same nine combinations of disk and background motion used in the perception experiments, subjects were also presented with stationary disks on a stationary background. These disks were presented approximately at the positions at which moving disks (of three different conditions) were hit. These positions depend on the $R T$ and $M T$ of the subject, and were therefore determined during the experiment. The stationary disks enabled us to evaluate the influence of target position on the movement of the hand. Combinations of disk direction and background velocity were presented in random order, so that subjects could never predict the following stimulus. Subjects could rest as often as they liked by simply not moving the rod back to the starting area. The experiment consisted of 15 trials for each of the 12 conditions.

\subsection{Analysis of movement data}

The velocity component perpendicular to the screen was calculated by numerical differentiation of the position data, without any filtering or smoothing. The beginning and end of the movement were determined by a threshold of $0.1 \mathrm{~m} / \mathrm{s}$ for this velocity. Trials were excluded from further analysis if the movement did not end on the screen, if infrared markers were hidden from view for more than $30 \mathrm{~ms}$, or if either the $R T$ or the $M T$ was more than $700 \mathrm{~ms}$. At least $85 \%$ of the trials fulfilled these criteria for all subjects.

The position data were filtered with a second-order digital Butterworth filter (Ackroyd 1973). The filter was applied in both forward and reverse directions to prevent phase shift. The effective cut-off frequency was $25 \mathrm{~Hz}$. Hereafter, we calculated (by linear interpolation) the displacement in the lateral and vertical directions as a function of the distance to the screen for each trial. These lateral and vertical displacements were averaged as a function of the distance to the screen to obtain average trajectories. 


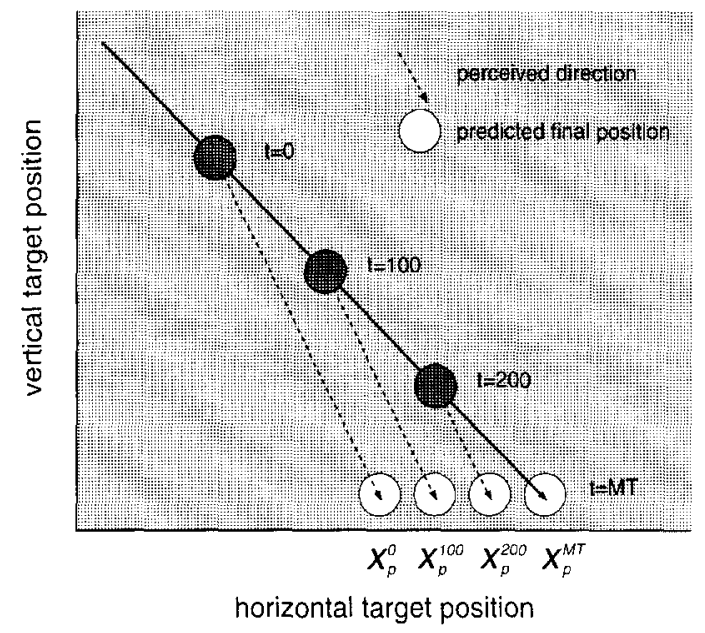

Fig. 2. Model for extrapolating a moving target's motion when the direction of motion is misperceived. The dashed lines show the perceived direction of motion. The extrapolation is given for three moments during the movement: $t=0$ is the time the hand starts to move; $x_{\mathrm{p}}^{0}$ is the extrapolated position at that instant. Continuous extrapolation moves the extrapolated position with velocity $v_{\mathrm{p}}=\mathrm{d} X_{\mathrm{p}}^{\mathrm{t}} / \mathrm{d} t$. The extrapolated position at movement time $\left(X_{\mathrm{p}}^{M T}\right)$ is of course the final position of the target

Motion of the background can influence the direction in which the hand moves according to the following reasoning. The vertical position of the target when hit will be the same for all conditions, because the vertical velocity is identical for all moving targets, and static targets appear at the vertical position at which the moving ones are hit. The actual lateral target position $x_{\mathrm{t}}$ at the time of the hit depends on the horizontal velocity $v_{t}$ of the target: $x_{\mathrm{t}}=x_{0}+(M T+R T) v_{\mathrm{t}}$. When anticipating the final lateral target position, the (horizontal) velocity of the background $v_{\mathrm{b}}$ can influence only the estimation of the part of the target motion that is yet to come (Fig. 2). If $t$ is the time after the reaction time $R T$, then we can describe the influence of the background on the estimated final target position as $G(M T-t) v_{\mathrm{b}}$. $G$ is the gain of the influence of the background: it is equal to 1 if a subject uses the direction of target motion relative to the background; it is equal to 0 if a subject ignores the background motion altogether. At a time $t$ after the reaction time $R T$, one can therefore expect the hand to be moving towards a position $x_{\mathrm{p}}$ on the screen:

$x_{\mathrm{p}}=x_{0}+(M T+R T) v_{\mathrm{t}}+G(t-M T) v_{\mathrm{b}}$

By comparing movements with and without background motion, we can estimate $G$ from the differences between the positions $x_{d}$ towards which these movements are directed at movement onset. Due to biomechanical factors and the subject's strategy, the hand will not move in a straight line to the target. Therefore, the positions $x_{\mathrm{d}}$ at which the hand is directed will be different from the predicted target position $x_{\mathrm{p}}$. As $x_{\mathrm{d}}$ is small with respect to the distance to the screen, we can write:

$x_{\mathrm{d}}=A+B\left[(M T+R T) v_{\mathrm{t}}+G(t-M T) v_{\mathrm{b}}\right]$ where $A$ and $B$ are factors which account for the systematic differences between the predicted target position $\left(x_{\mathrm{P}}\right)$ and the direction in which the hand starts to move $\left(x_{d}\right)$. As already mentioned, these systematic differences (the curvatures in the trajectories) are caused by the subject's strategy and by biomechanical factors.

To quantify the effect of background motion, we examined how a moving background influences the direction in which the hand starts to move. Note that (2) can only be used near movement onset, i.e. for small values of $t$, because during a movement the direction of movement is determined not only by $x_{\mathrm{p}}$, but also by the history of the movement (position, velocity). To determine the position on the screen towards which the trajectory was directed $\left(x_{\mathrm{d}}\right)$, we had to determine the tangent of the trajectory. The shortest time $t$ at which this could be determined accurately enough was about $70 \mathrm{~ms}$ after movement onset. At this time the hand was less than $1 \mathrm{~cm}$ from its starting position. The value of $G$ (and $A$ and $B$ ) was determined for each subject by a linear regression of the data of all trials to (2).

The disadvantage of this approach is that (2) only holds at movement onset. At that moment, however, the direction of motion is not easy to measure. To determine the position $x_{\mathrm{p}}$ towards which the movement is directed at other moments during the movement, we have to be more explicit about the biomechanical parameters $A$ and $B$. To do this, we need a model of the arm movement.

\subsection{Modelling approach}

The aim of our model is very modest: it is to describe differences between hand movements towards different (moving) targets in relation to the underlying differences in visual information. All movements in our experiment have more or less the same shape and velocity profile: they are all directed forwards with about the same vertical component of the movement; the only (relatively small) variations between conditions are in the lateral component of the movement. In our model we ignore the other components of the motion, and concentrate on the small lateral differences between the conditions. This allows us to approximate the highly non-linear motor system by a linear model. For this approximation, we must define a point in the variable space around which the system is assumed to be linear. We take the average of all movements as a reference and regard all movements as (lateral) deviations from this reference movement. Thus, only these lateral deviations are modelled by the simple (linear) system.

A simple linear system which can describe movements of a limb is a mass-spring model, as introduced in the field of motor control by Feldman (1966). Although one can question its use in describing the (non-linear) fast goal-directed movements themselves (Smeets 1992), it is very well suited to describe small variations between movement conditions. In the mass-spring model one can distinguish between the mechanical parameters, and variables describing the target position.

In our implementation of the model, we assume that changes in visual information about the target's current 
position cause changes in the predicted position $x_{p}$ of the target, leaving the mechanical parameters unchanged. The values of the mechanical parameters were therefore determined from experiments in which there is no doubt about the value of $x_{\mathrm{p}}$ : movements towards stationary targets.

We can now evaluate the influence of visual information by estimating the changes in the predicted target position $x_{\mathrm{p}}(t)$ as a function of time. For this estimation, we assume that the changes in the predicted target position $x_{\mathrm{p}}(t)$ are caused by extrapolation errors. As an error in the perceived direction leads to a linear change in the expected target position (Fig. 2), we assume that the predicted target position moves at a fixed velocity $v_{\mathrm{p}}$, starting at a position $x_{\mathrm{p}}^{0}$. Using three parameters (mass $m$, viscosity $b$ and stiffness $k$ ) to describe the mechanics, the differential equation for the lateral position of the hand $x$ (relative to the reference movement) is therefore:

$m \ddot{x}+b \dot{x}+k x=k\left(x_{\mathrm{p}}^{0}+v_{\mathrm{p}} t\right)$

The way to solve this kind of equation can be found in many textbooks on mathematics, and is reproduced in Smeets and Brenner (1995). Choosing one parameter equal to 1 simplifies the calculations, without changing the resulting solution; we chose $m=1 \mathrm{~kg}$. After applying the boundary conditions $(x(0)=\dot{x}(0)=0)$ and substituting $x_{\mathrm{p}}^{0^{\prime}}=x_{\mathrm{p}}^{0}-b v_{\mathrm{p} /} / k$ and $\omega=\sqrt{\left(k-b^{2} / 4\right)}$, the solution to (3) becomes:

$$
\begin{aligned}
x(t)=v_{\mathrm{p}} t+x_{\mathrm{p}}^{0^{\prime}}-x_{\mathrm{p}}^{0^{\prime}} e^{-b r / 2} & \\
& {\left[\cos (\omega t)+\left(\frac{b}{2 \omega}+\frac{v_{\mathrm{p}}}{x_{\mathrm{p}}^{0^{\prime}} \omega}\right) \sin (\omega t)\right] }
\end{aligned}
$$

To obtain estimates for the parameters, we fitted this equation to the experimental data (Levenberg-Marquardt method: Press et al. 1987). We did so in two steps. The mechanical parameters $k$ and $b$ (and thus the eigenfrequency $\omega$ of the system) were determined by fitting (4) to the movements towards the stationary targets (for each subject separately, substituting $v_{\mathrm{p}}=0$ and $x_{\mathrm{p}}^{0}=$ actual target position). Using these values for $k$ and $b$, (4) was fitted in the second step to the experimental data for all target conditions, yielding for each subject and each target condition an estimate for $v_{\mathrm{p}}$ and $x_{\mathrm{p}}^{0}$. If all the deviations in trajectories were caused by extrapolating with the (wrong) perceived direction, then $x_{\mathrm{p}}^{0}$ and $v_{\mathrm{p}}$ should be predictable from the perception experiments (Fig. 2). Besides presenting values of the initial predicted position $x_{\mathrm{p}}^{0}$, we will also present values of the prediction error $E_{\mathrm{p}}^{0}$. This is the difference between the initial and the final predicted target position: $E_{\mathrm{p}}^{0}=x_{\mathrm{p}}^{0}-x_{\mathrm{p}}^{M T}$. A zero prediction error does not indicate that the hand initially moves in the direction of the target's position at the time of the hit, but it indicates that the hand initially moves in the same direction as it would move towards a stationary target at that position.

\section{Results}

\subsection{Perception experiments}

All subjects showed similar responses in the perception experiments. The average settings are shown in Fig. 3.
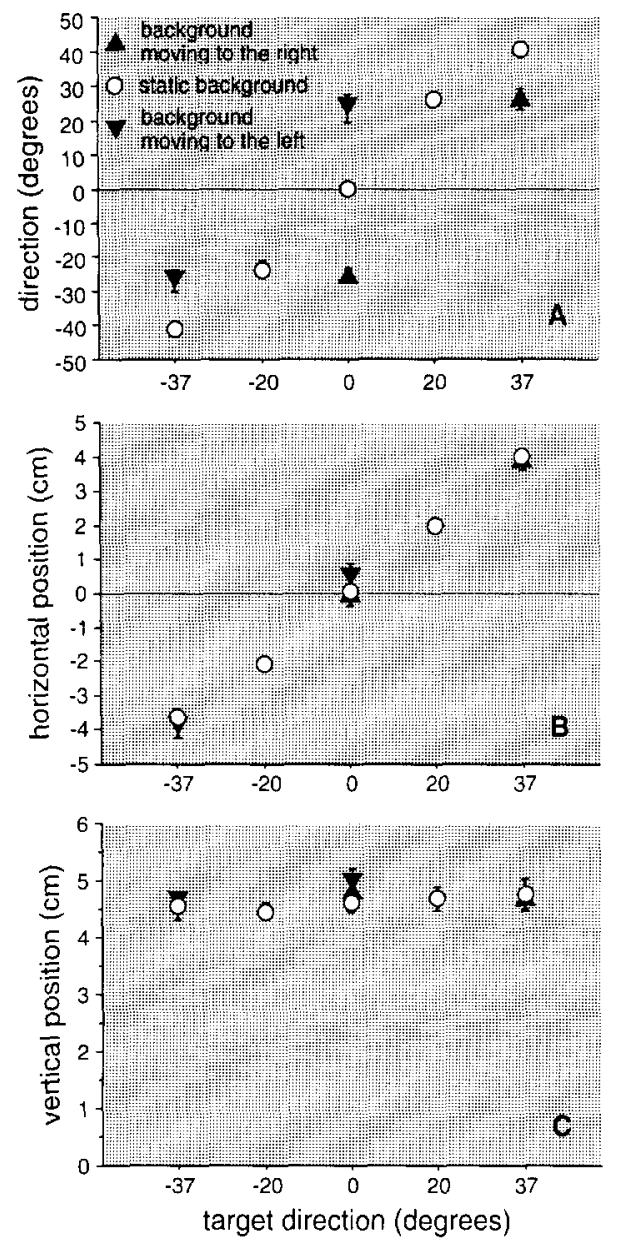

Fig. 3. The results of the perception experiments, averaged over all ten subjects. Symbols indicate the background motion; error bars indicate the inter-subject standard error of the mean. When the background moved, its speed was equal to the horizontal component of the motion of a target moving $37^{\circ}$ from vertical. A The results for matching the perceived direction. Motion of the background had a highly significant effect on the perceived direction of motion $(P<0.001)$. B, C The results for matching the perceived position. Motion of background had no significant $(P>0.05)$ effect on the perceived positions

Subjects made systematic errors in indicating the direction of target motion. With a static background, the angle of motion was slightly $(15 \%)$ over estimated. The motion of the background had a clear effect on the perceived direction. The perceived direction was about midrange between the actual direction and the direction relative to the background: the background motion influenced the perceived angle of motion with a gain of $0.5 \pm 0.1$. An ANOVA revealed that for all directions of target motion in which the background could move, the background had a highly significant $(P<0.01)$ effect on the perceived direction of motion.

The position at which the target disappeared was perceived quite accurately. The $4.4 \mathrm{~cm}$ vertical motion was (on average) perceived as $4.7 \mathrm{~cm}$, an overshoot of about $10 \%$. There was no systematic effect of target direction or background motion on this overshoot. As might be expected on the basis of the vertical overshoot, 
the horizontal movements were also (on average) over estimated by about $10 \%$. In no case did background motion have a significant effect on the perceived position (ANOVA, $P>0.05$ ). A possible small $(0.1-0.3 \mathrm{~mm}$; not significant) influence of background motion could be attributed to extrapolation on the basis of the perceived direction during the overshoot.

On the basis of these results, one can make a prediction for the motor experiments. Assuming that the perceived direction of motion is used to predict the target position at the time of the hit, we would expect considerable errors in starting direction. We expect such errors, and adjustments during the movements-as the amount of required extrapolation decreases - to be visible in the arm movements towards moving targets.

\subsection{Goal-directed movements}

The reaction time $(300 \pm 50 \mathrm{~ms}$; mean of all subjects \pm standard deviation between subjects) and movement time ( $280 \pm 55 \mathrm{~ms}$ ) differed considerably between subjects, but showed no systematic relationship with the motion of either the target or the background. The arm accelerated continuously until reaching the screen, reaching a maximum velocity of $2.7 \pm 0.8 \mathrm{~m} / \mathrm{s}$. The direction of movement of the hand just after movement onset depended systematically on the motion of the background: if the background moved leftward, the hand aimed further to the right, and vice versa. To quantify this effect, we estimated the gain of the effect of the background motion

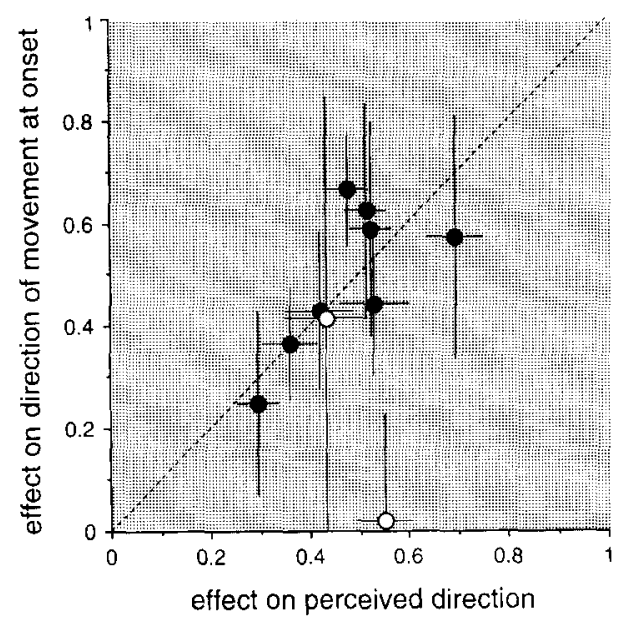

Fig. 4. The effect of background motion on the perceived direction of motion and on the direction in which the hand starts moving. Each data point is the average for one subject; the horizontal and vertical bars give the standard error. The open symbols represent the two subjects whose data were discarded from further analysis on the basis of the criteria mentioned in the text. Abscissa: the (dimensionless) gain $G$ obtained from the direction of hand movement near movement onset (2). Ordinate: the effect of horizontal background motion on the perceived direction as a fraction of the effect that an identical horizontal motion of the target would have on the perceived direction. The dashed line is the relation one would expect if the perceived direction of motion is used to direct the hand. For the subjects indicated by filled symbols, the effect on the direction of movement never differed significantly from the perceptual effect $(t$-test, $P>0.05)$ (see Sect. 2.6). In Fig. 4 we compare the effect of background motion on the direction of arm movement $[G$ according to (2)] with the effect on the perceived direction. Apart from two subjects, all subjects showed similar clear effects in both experiments. We can conclude that most subjects use the perceived direction in estimating the target position when hitting a moving object.

An overview of one subject's trajectories (corrected for the average curvature) is given in Fig. 5A. The global difference in shape between the trajectories in different target conditions was the same for all subjects. On average, subjects missed the centre of the target by $1.3 \pm 0.8 \mathrm{~cm}$. There were some systematic biases: on average, subjects hit the screen slightly $(1 \mathrm{~mm})$ below the target. Targets on the right were hit slightly to the right of their actual position, whereas targets on the left were hit slightly to the left. This position-dependent bias did not differ systematically between the stationary and moving targets. On average, the moving targets were hit $2 \mathrm{~mm}$ further to the right than the stationary targets. Motion of the background had no systematic effect on the hit error. As even the trajectories to static targets are curved, the direction of the hand movement cannot directly tell us how the visual information is used. To answer this question, we need a model which can deal with the curvature
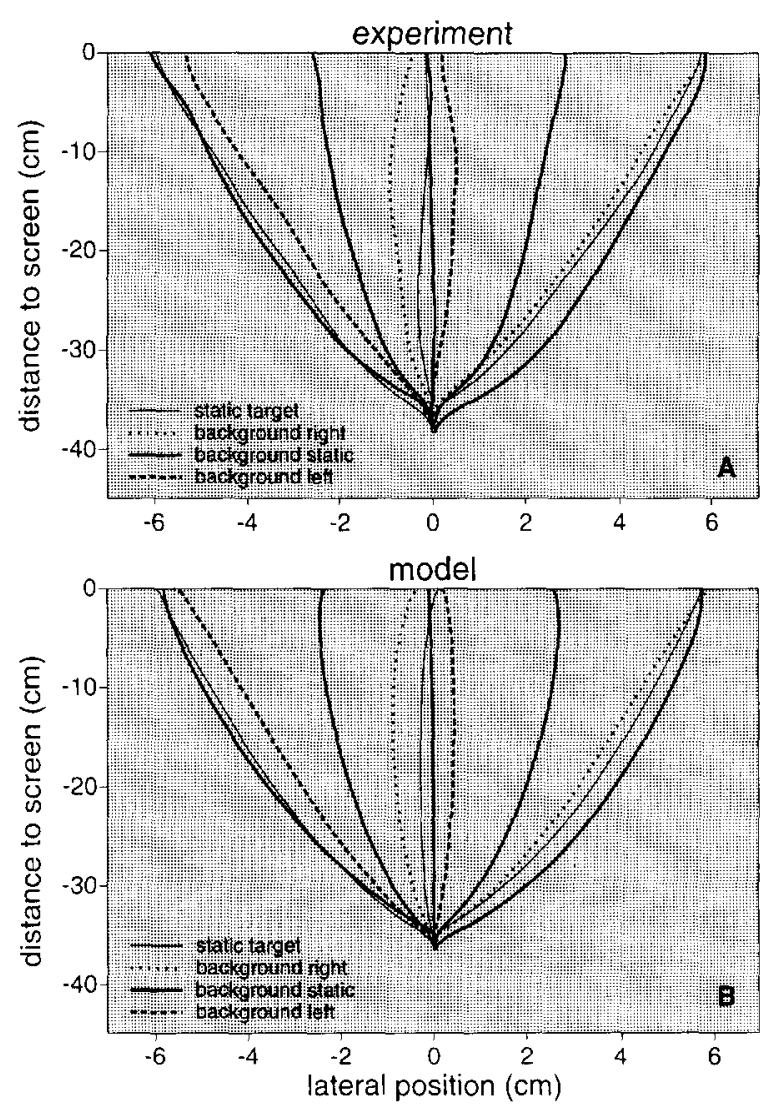

Fig. 5. Top view of the differences between trajectories of one subject's hand. Each trace is the average of 12-15 trials for one target condition. The subject's average lateral movement (averaged over all 171 trials) is subtracted from each trajectory (see Sect. 2.7). A The experimental data. B The best fit of the model, obtained as described in Sect. 2.7 
of the trajectories. The next section will describe the results obtained by analysing the experimental data with the model presented in Sect. 2.7.

\subsection{Model calculations}

One of the assumptions of the model is that movements to stationary targets and to moving targets are made with the same mechanical parameters. Before analysing the experiments with our model, we checked whether all subjects satisfied this requirement. To do so, we looked at the vertical component of the movement. As the stationary disks were presented at the positions at which moving disks were hit, the vertical component of the movements should be equal for all conditions. Therefore, different vertical trajectories towards stationary and moving targets indicate that different strategies are used. For each subject, we tested whether the vertical position at $20 \mathrm{~cm}$ from screen differed significantly $(P<0.05)$ between trajectories towards static and moving targets. For two subjects (open symbols in Fig. 4), the movements towards stationary targets started systematically towards lower positions than movements towards moving targets. These two subjects (who also had longer reaction times and higher maximum velocities than the other eight) were excluded from further analysis. These two were the only subjects for whom background motion did not influence the direction of movement at onset by more than 1 standard error (Fig. 4).

For the other eight subjects, the mechanical parameters $k$ and $b$ were determined from a fit of the model to the movements towards stationary targets. In accordance with the variations in the maximum velocities of the hand, these mechanical parameters differed substantially between subjects. The average values $(k=120 \pm 50 \mathrm{~N} / \mathrm{m}$ and $b=14 \pm 5 \mathrm{Ns} / \mathrm{m}$ ) seem reasonable for the stiffness and viscosity of a human arm (Flash 1987). Subjects with high stiffness also had high viscosity, resulting in considerably smaller differences between subjects in the eigenfrequency than in $k$ or $b$. The eigenfrequency was $\omega=8.2 \pm 1.7 \mathrm{rad} / \mathrm{s}$. This value corresponds to a period of $0.77 \mathrm{~s}$ for oscillations of the arm.

We used (4) to obtain estimates of the predicted target position for each condition and subject. For each subject, we substituted in (4) the mechanical parameters which we obtained from that subject's trajectories towards stationary targets. The predicted target position at the beginning of the movement (the initial predicted position $x_{\mathfrak{p}}^{0}$ ) and the velocity with which the predicted position changes during the hit $\left(v_{\mathrm{p}}\right)$ were obtained by subsequently fitting the equation to the data for each target condition. The fitted trajectories are shown for one subject in Fig. 5B. The trajectories towards the static targets are fits of (4) with $v_{\mathrm{p}}=0$ and $x_{\mathrm{p}}^{0}=$ the actual target position. The curved shapes of these trajectories are caused by the mass-spring behaviour of the system. The model trajectories match the measured trajectories very well.

The trajectories towards moving targets on a static background have approximately the same curvature as those towards the static targets. This indicates that $v_{\mathrm{p}}$ is small: i.e. the estimated target position $x_{\mathrm{p}}$ did not change much during the movements. Motion of the background changes the curvatures in comparison with the condition without background motion. This change corresponds with an initial predicted target position $x_{\mathbf{p}}^{0}$ that is shifted in the direction opposite to that in which the background is moving, followed by 'motion' of the predicted target position (with a velocity $v_{\mathrm{p}}$ ) in the same direction as the motion of the background.

The resulting values for the fit parameters of all subjects are plotted in Fig. 6. In Fig. 6A we can see that
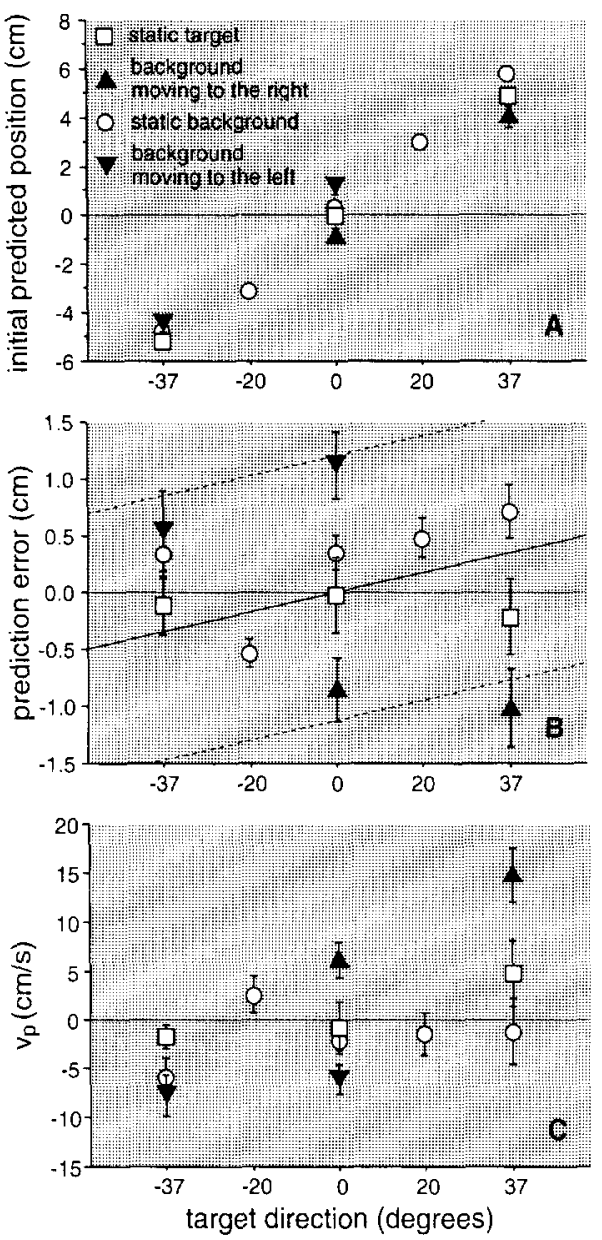

Fig. 6. Parameters describing the prediction of the target's final position [obtained by fitting the model (4) to the measured trajectories]. The parameters were obtained separately for each subject and target condition, and subsequently averaged. Error bars indicate the intersubject standard error (eight subjects). Symbols are as in Fig. 3, with the squares representing the stationary targets on a static background (not used in the perception experiments). For all three model parameters, motion of the background had a highly significant $(P<0.01)$ effect for targets moving in the directions $0^{\circ}$ and $37^{\circ}$. For targets moving in the $-37^{\circ}$ direction, the effect was not significant $(P>0.05)$. A The initial predicted position $\left(x_{\mathrm{p}}^{0}\right)$. B The prediction error $\left(E_{\mathbf{p}}^{0}\right)$ : the difference between the target's initial predicted position and its actual position when the screen is hit. The symbols are the values obtained by fitting the model to the trajectories. The lines show the error one would predict if subjects aim towards a position that is extrapolated on the basis of the perceived target direction. Continuous line, background static; dashed lines, background moving. The slope is caused by a systematic overestimation of the angle of motion (relative to the vertical). The shift between the lines is caused by the systematic influence of background motion. $\mathbf{C}$ The velocity with which the predicted target position changes $\left(v_{\mathrm{p}}\right)$ 
the initial predicted position varies with the direction of target motion. Moreover, motion of the background has a systematic influence on the initial prediction: it is about $1 \mathrm{~cm}$ further to the right when the background is moving to the left, and about $1 \mathrm{~cm}$ further to the left when the background is moving to the right (triangles in Fig. 6A). The effect of background motion is about $25 \%$ of the effect of real target motion with the same horizontal velocity. According to (1), with $R T \approx M T$ and $t=0$, it follows that the gain of the effect of background motion is $G \approx 0.5$. The model thus replicated the finding of Fig. 4: the perceptual effect of motion of the background is the same as the effect on the hand movements.

The model reveals more about how the hand is directed towards the target. To see this more clearly, we show the prediction error as a function of the direction of target motion in Fig. 6B. Apart from the effect of background motion, we now also see a small but systematic effect of the direction of target motion when the background is static. The hand is initially aimed to the right of targets moving rightward and to the left of targets moving leftward. This effect is consistent with the systematic over estimation of direction in the experiment on the perceived direction of motion (Fig. 3A). The continuous line in Fig. 6B is the effect predicted from the perceived direction of motion. It is determined by extrapolating during the movement time with the perceived direction of motion from the target's position at reaction time. Similarly, with a gain for the effect of background motion on the perceived direction of motion of 0.5 , we expect an additional error in the prediction of $E_{\mathrm{p}}^{0}=0.5 v_{\mathrm{b}} M T$ (predictions shift to the dashed lines in Fig. 6B). The data are consistent with the assumption that initial error is determined by errors in the perceived direction of motion. We conclude that the fit parameter $x_{p}^{0}$ in our model is a good representation of the position towards which the hand is directed at movement onset.

In Fig. 6C we present the velocity with which the prediction of the position of the target changes during the movement, for the different directions of target motion. Motion of the background influences this parameter systematically. As one would expect, the velocity at which the predicted final position changes is inversely proportional to the error in the initial prediction. The correlation between prediction error and velocity can be compared with what one would expect on the basis of the simple model shown in Fig. 2 . If we assume that the prediction of the target position is adjusted continuously, the error in predicting the target position will decrease to zero during the movement. We therefore expect the velocity with which the predicted target position changes $\left(v_{\mathrm{p}}\right)$ to be equal to the quotient of the prediction error $\left(E_{\mathrm{p}}^{0}\right)$ and movement time $(M T)$.

We expect the same relation between $v_{\mathrm{p}}$ and $E_{\mathrm{p}}^{0} / M T$ for all subjects and all conditions of background motion. In Fig. 7 we show $v_{\mathrm{p}}$ as a function of $E_{\mathrm{p}}^{0} / M T$ for each subject and each moving target condition. The velocity of the predicted target position is indeed correlated with the prediction error per movement time $\left(r^{2}=0.7\right)$. This correlation does not only hold when we introduced an initial error by moving the background (triangles), but also for

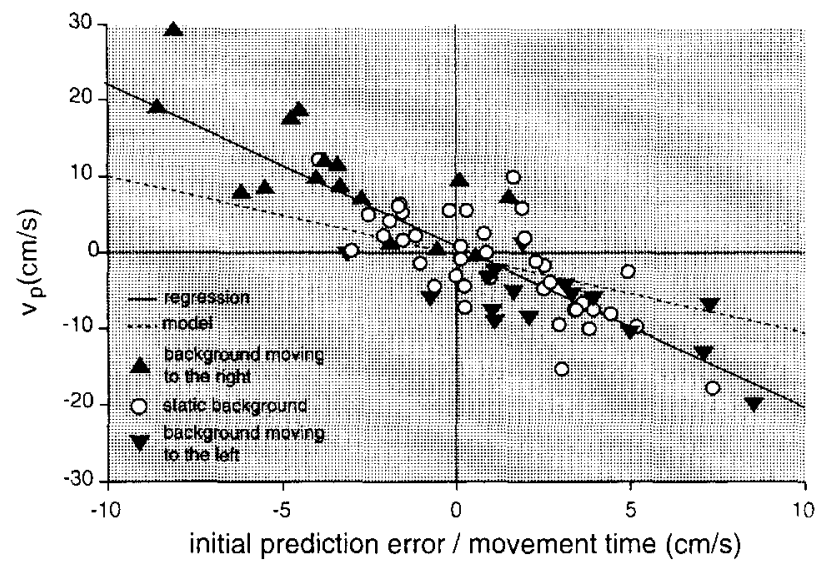

Fig. 7. The relationship between the ratio of prediction error and movement time $\left(E_{\mathrm{p}}^{0} / M T\right)$ and the velocity with which the predicted target position changes $\left(v_{\mathrm{p}}\right)$. Each point represents the parameters for one subject and target condition. The continuous line is a linear regression of the data; the dashed line represents the expected motion of the predicted target position as a result of continuously updating the predicted target position (see Fig. 2)

the other target conditions (circles). However, the slope of the relation is 2.1 , significantly $(P<0.01)$ different from the slope of 1.0 predicted by our model. The relevance of this finding is discussed in the next section.

\section{Discussion}

We have shown that a visual illusion which influences the direction in which an object is perceived to move changes the direction in which one's hand starts to move towards that object. The variations in the trajectory of the hand can be modelled by a spring-like attraction to a (constantly changing) prediction of the final target position. Neither the idea of a continuously updated internal representation of a target's position (van Sonderen et al. 1988), nor the idea of continuous use of this information for the control of an ongoing movement (van Sonderen et a1. 1989) is new. What is new is the attempt to determine the initial prediction error (and the shift in the expected final target position as this error is corrected) from the movement data, and to compare this with errors in perception.

The modelling approach we use is different from most other models. Most models attempt to test principles of motor control. Examples of such principles are the use of internal representations (e.g. van Sonderen and Denier van der Gon 1990; Gerdes and Happee 1994), equilibrium points (e.g. Feldman 1966; Latash 1994) or minimization of jerk (Flash 1987; Hoff and Arbib 1992). Our model is not an attempt to show how motor control works. We do not model the movements themselves, but only compare the differences between movements. Nevertheless, the results can be compared with parameters of some other models. The parameter $x_{\mathrm{p}}$ in our model corresponds to the internal representation of target position ('trace') in the model of van Sonderen and Denier van der Gon (1990), and to the equilibrium point 
in studies based on the equilibrium point hypothesis (Flash 1987; Latash and Gottlieb 1992). In order to describe the movements themselves (or if the differences between movements become larger), the linear approximation that our model is based on will not hold. At that moment, assumptions on the principles of motor control have to be made: non-linearities are introduced into the model (van Sonderen and Denier van der Gon 1990) or a non-linear movement of the equilibrium point is assumed (Latash and Gottlieb 1992). We restrict ourselves to small differences for which the linear approximation does hold.

The main result of this study is that we show that we can predict the initial errors in directing the hand towards the target on the basis of errors in the perceived direction of target motion. The initial part of the trajectories of the hand was not only in accordance with the influence that background motion has on the perceived direction of motion, but also with 'spontaneous' systematic errors in the perceived direction of motion of the target (Fig. 6B). We observed systematic errors for targets on static backgrounds: an overestimation of angles relative to the vertical by up to $7^{\circ}$. These errors are comparable to those reported by de Graaf et al. (1991).

The influence of background motion on the direction in which the hand started to move was evident from the straightforward analysis of the data [(2), Fig. 4]. The analysis with the linear second-order model confirmed this finding [(4), Fig. 6B]. This model revealed the influence of the above-mentioned over estimation of the direction (relative to the vertical) of target motion on the direction in which the hand started to move. Despite initial systematic errors in the prediction of the target position of more than $1 \mathrm{~cm}$, subjects hit (on average) within a few millimetres of the targets. How are these initial errors corrected for during the movement?

Our suggestion is that the predicted position of the target gradually moved as real changes in target position replaced the anticipated change. Only the latter was misled by background motion (Fig. 2). According to this suggestion, information obtained before the start of the movement can change the trajectory just after movement onset. This suggestion cannot explain the data (Fig. 7). The position at which subject anticipated to hit the target moved twice as fast as expected. Reversing the argument: if the predicted target position were obtained in the suggested manner (Fig. 2), the linear second-order model would predict that subjects should hit about $0.8 \mathrm{~cm}$ behind the target. In fact, they hit slightly ahead of the target. Small changes in the model, such as introducing a delay, cannot remove this discrepancy between the model predictions and the experiment.

This discrepancy can be interpreted in two ways. The first is that subjects interpret the change in the extrapolated target position as a change in its direction. Thus, the estimated direction of motion changes during the trial. A second possibility is that one of the assumptions of the model is not valid. For instance, the variations in the mechanical parameters during the movement could be too large to justify the linear approximation in (3). If this is the case, we can expect that the value obtained for $v_{\mathrm{p}}$ has no direct relation to changes in the predicted position of the target. To discuss this second possibility, we will take a closer look at the assumptions we made in our model.

We made two assumptions when determining the mechanical parameters $k$ and $b$. The first is that subjects used the same movement strategy (and thus $k$ and $b$ were the same) in movements towards stationary and moving targets. The second is that the mechanical parameters and the perceived position of the static targets do not change during the movement. The way we were able to evaluate the first assumption has been described in Sect. 3.3. This resulted in the rejection of the data of two (of the ten) subjects. The second assumption is evaluated by examining the consistency within the model. We examined whether the model yields a zero prediction error $E_{\mathrm{p}}^{0}$ and zero velocity $v_{\mathrm{p}}$ for the stationary targets if the predicted target position was not fixed (as we did to determine $k$ and $b$ ) but was allowed to move. The results of this analysis (a fit of the trajectories with $v_{\mathrm{p}}$ and $x_{\mathrm{p}}^{0}$ as free parameters) are shown by the open squares in Fig. 6 . The squares in Fig. $6 \mathrm{~B}$ are the calculated prediction errors for the movements towards stationary targets. These prediction errors are indeed close to zero. The velocity of the predicted target position $v_{\mathrm{p}}$ (Fig. 6C) does seem to depend on the position of the stationary target, although this was not significant $(P>0.05)$. This behaviour could be caused by a dependency of $k$ and $b$ on the posture of the arm, which is quite likely (Flash 1987; Flash and Mussa-Ivaldi 1990). We must, therefore, be cautious in interpreting values of $v_{\mathrm{p}}$, and cannot conclude conclusively that the direction of motion our subjects used to extrapolate the target's motion changed during their arm movement.

The most important conclusion from this study is that an arm movement to a moving target starts towards a position that is obtained by extrapolating from the actual position of the target using the perceived direction of target motion. We succeeded partially in attaining the second goal of this paper: to model the continuous use of visual information in guiding arm movements. The initial part of the movement was consistent with a linear model of the mechanics of the arm, which makes continuous use of visual information on the target's position. The model could not explain all the corrections that were made during the rest of the trajectory in order to compensate for the initial errors. Further research is required to reveal whether our simple hypothesis on motion of predicted position (Fig. 2) is wrong or whether the mechanical parameters of the arm change too much during the movements to neglect such changes.

\section{References}

Ackroyd MH (1973) Digital filters. Butterworth, London

Brenner E, Smeets JBJ (1994a) Different frames of reference for position and motion. Naturwissenschaften $81: 30-32$

Brenner E, Smeets JBJ (1994b) Why we hit slow targets more gently. J Physiol (Lond) 479P:53-54

Feldman AG (1966) Functional tuning of the nervous system during control of movement or maintenance of a steady posture. II. Controllable parameters of the muscle. Biofizika 11:498-508 
Flash $\mathrm{T}$ (1987) The control of hand equilibrium trajectories in multijoint arm movements. Biol Cybern 57:257-274

Flash T, Mussa-Ivaldi F (1990) Human arm stiffness characteristics during the maintenance of posture. Exp Brain Res 81:315-326

Gerdes VGJ, Happee R (1994) The use of an internal representation in fast goal-directed movements: a modelling approach. Biol Cybern $70: 513-524$

Graaf JB de, Sittig AC, Denier van der Gon JJ (1991) Misdirections in slow goal directed arm movements and pointer setting tasks. Exp Brain Res 84:434-438

Hoff B, Arbib MA (1992) A model of the effects of speed, accuracy, and perturbation on visually guided reaching. In Camminiti R, Johnson PB, Burnod Y (eds) Control of arm movements in space: neurophysiological and computational approaches. Springer, Berlin Heidelberg New York, pp 285-306

Latash ML (1994) Reconstruction of equilibrium trajectories and joint stiffness patterns during single-joint voluntary movements under different instructions. Biol Cybern 71:441-450

Latash ML, Gottlieb GL (1992) Virtual trajectories of single-joint movements performed under two basic strategies. Neuroscience $47: 357-365$

Lee DN, Young DS (1986) Gearing action to the environment. In Heuer $\mathrm{H}$, Fromm $\mathrm{C}$ (eds) Generation and modulation of action patterns. Springer, Berlin Heidelberg New York, pp 217-230
Peper L, Bootsma RJ, Mestre DR, Bakker FC (1994) Catching balls: how to get the hand to the right place at the right time. J Exp Psychol Human Percept Perform 20:591-612

Press WH, Flannery BP, Teukolsky SA, Vetterling WT (1987) Numerical recipes in C: the art of scientific computing. Cambridge University Press, Cambridge

Smeets JBJ (1992) What do fast goal-directed movements teach us about equilibrium-point control? Behav Brain Sci 15:796-797

Smeets JBJ, Brenner E (1995) Perception and action based on the same visual information: distinction between position and velocity. J Exp Psychol Human Percept Perform 21:19-31

Sonderen JF van, Denier van der Gon JJ (1990) A simulation study of a programme generator for centrally programmed fast two-joint arm movements: responses to single- and double-step target displacements. Biol Cybern 63:35-44

Sonderen JF van, Denier van der Gon JJ, Gielen CCAM (1988) Conditions determining early modification of motor programmes in response to changes in target location. Exp Brain Res $71: 320-328$

Sonderen JF van, Gielen CCAM, Denier van der Gon JJ (1989) Motor programmes for goal-directed movements are continuously adjusted according to changes in target location. Exp Brain Res $78: 139-146$ 